\title{
RESULTADOS DA CIRURGIA DE RESERVATÓRIOS ILEAIS EM PACIENTES COM DOENÇA DE CROHN
}

\author{
Maria de Lourdes Setsuko AYRIZONO, Luciana Rodrigues MEIRELLES, Raquel Franco LEAL, \\ Cláudio Saddy Rodrigues COY, João José FAGUNDES e Juvenal Ricardo Navarro GÓES
}

\begin{abstract}
RESUMO - Racional - Retocolectomia total e reservatório ileal com anastomose ileoanal constitui o procedimento de escolha para doentes com retocolite ulcerativa inespecífica que requerem cirurgia. Entretanto, alguns doentes por ela acometidos podem desenvolver características compatíveis com doença de Crohn, com conseqüente falência do reservatório. Objetivo - Avaliar a evolução tardia dos doentes com reservatórios ileais cujo diagnóstico definitivo foi doença de Crohn. Métodos - Entre fevereiro de 1983 a março de 2007, 151 doentes do Grupo de Coloproctologia do Hospital das Clínicas da Universidade Estadual de Campinas, SP, foram submetidos a retocolectomia total e reservatório ileal, sendo 76 por retocolite ulcerativa inespecífica. Destes, 11 (14,5\%) evoluíram como doença de Crohn, com diagnóstico histopatológico confirmado em 9: 1 no espécime da retocolectomia, 2 na proctectomia, 2 em segmentos de intestino delgado, 3 em reservatórios ileais, sendo 2 em biopsias e 1 no reservatório ressecado, e 1 em material de abscesso perianal. Oito doentes (72,7\%) eram mulheres e a média de idade foi de 30,6 (18-65) anos. Resultados - Todos tinham diagnóstico pré-operatório de retocolite ulcerativa inespecífica e cinco foram operados inicialmente por megacólon tóxico. O tempo médio entre a confecção do reservatório ileal e a manifestação da doença de Crohn foi de 30,6 meses. Ileostomia de proteção não foi fechada apenas em um doente que apresentou fístula do reservatório no enema opaco e, posteriormente, abscesso perianal extenso e recidiva da doença de Crohn na alça aferente do reservatório. No seguimento tardio, três doentes evoluíram com fístulas perianais e perineais complexas, sendo associada à fístula reservatório-vaginal em uma delas. Todos necessitaram de nova derivação e o reservatório foi excisado em um deles devido à persistência da sepse pélvica. Uma outra apresentou fístula do reservatório para o intróito vaginal, corrigida com cirurgia de avanço mucoso. Houve também progressão da doença para o íleo proximal em três doentes. Nos outros três, a recidiva da doença foi no reservatório ileal, com boa resposta ao tratamento clínico. Atualmente, com seguimento médio de 76,5 meses, todos os doentes estão assintomáticos, sendo que quatro estão com derivação e sete com reservatórios funcionantes, estes apresentando 6-10 evacuações ao dia e continência praticamente normal. Conclusão - Recidiva da doença de Crohn após retocolectomia e reservatório ileal é relativamente freqüente, com ocorrência de complicações como abscessos, fístulas e estenoses. Entretanto, reservatórios ileais com doença de Crohn e mantidos, podem ser compatíveis com função satisfatória.
\end{abstract}

DESCRITORES - Doença de Crohn. Proctocolite. Bolsas do colo. Bolsas do íleo.

\section{INTRODUÇÃO}

Desde a descrição inicial por PARKS e NICHOLLS ${ }^{(16)}$, a retocolectomia total com reservatório ileal e anastomose ileoanal tem se tornado o procedimento de escolha para doentes com retocolite ulcerativa inespecífica (RCUI) que requerem cirurgia, e com polipose adenomatosa familiar (PAF) com acometimento do reto. O procedimento remove o intestino doente, propicia continência e função anal satisfatória e evita a necessidade de uma ileostomia definitiva. Esta cirurgia é como regra, na maioria das vezes, contra-indicada na doença de Crohn (DC) pelo elevado risco de recurrência no reservatório com o potencial de se desenvolver fístulas, estenoses, deiscências de suturas e formação de abscessos ${ }^{(2,3,5,10,21)}$.
Entretanto, em alguns casos, a distinção entre RCUI e DC é difícil, mesmo quando o patologista dispõe de toda a peça da retocolectomia ${ }^{(24)}$.

O objetivo deste estudo foi avaliar os doentes operados de reservatório ileal por RCUI e que evoluíram posteriormente com diagnóstico de DC.

\section{MÉTODOS}

Entre fevereiro de 1983 e março de 2007, 151 doentes foram submetidos a retocolectomia total e reservatório ileal, no Serviço de Coloproctologia da Disciplina de Moléstias do Aparelho Digestório da Universidade Estadual de Campinas, SP (UNICAMP). Setenta e seis tinham RCUI, 72 PAF, 1 DC, 1 carcinoma metacrônico

\footnotetext{
Grupo de Coloproctologia da Disciplina de Moléstias do Aparelho Digestório do Departamento de Cirurgia e Departamento de Anatomia Patológica Faculdade de Ciências Médicas - Universidade Estadual de Campinas, São Paulo, SP.

Correspondência: Dra. Maria de Lourdes Setsuko Ayrizono - Rua Emília de Paiva Meira, 123 - apt. 51 - Bairro Cambuí - 13025-040 - Campinas, SP.

E-mail: luayrizono@terra.com.br
} 
de cólon direito após cirurgia de abaixamento e outro, adenoma do reto pós-colectomia total e anastomose ileorretal. Dos doentes operados como tendo RCUI, 11 (14,5\%) tiveram o diagnóstico mudado para DC. Em nove deles houve confirmação histológica, sendo uma no espécime da retocolectomia, dois na proctectomia, dois em segmentos de intestino delgado excisados no pós-operatório tardio devido à recidiva da doença, três em reservatórios ileais (duas em biopsias e uma no reservatório ressecado) e uma em material de borda de abscesso perianal (Figura 1). Oito doentes $(72,7 \%)$ eram mulheres e a idade variou de 18 a 65 anos, com média de 30,6.

\begin{tabular}{|lccl|}
\hline Doente & Idade & Sexo & Diagnóstico DC \\
\hline 1 & 23 & F & Sem confirmação histológica \\
2 & 29 & F & Retocolectomia \\
3 & 21 & F & Enterectomia \\
4 & 18 & M & Enterectomia \\
5 & 40 & M & Reservatório ileal \\
6 & 28 & F & Biopsias reservatório ileal \\
7 & 34 & F & Sem confirmação histológica \\
8 & 20 & F & Biopsias reservatório ileal \\
9 & 65 & F & Abscesso perianal \\
10 & 38 & M & Proctectomia \\
11 & 21 & F & Proctectomia \\
\hline
\end{tabular}

FIGURA 1. Caracterização da casuística

\section{RESULTADOS}

Previamente às cirurgias, todos tinham biopsias confirmando diagnóstico de RCUI e cinco doentes foram inicialmente submetidos a colectomia por megacólon tóxico. O tempo médio entre a confecção do reservatório ileal e a manifestação clínica da DC foi de 30,6 meses, variando de 6 a 80 meses.

Um doente não teve seu estoma de proteção fechado devido à fístula do reservatório no enema opaco de controle. Apresentou também, após 13 meses da cirurgia, extenso abscesso perianal cuja biopsia local revelou tratar-se de DC e o exame endoscópico demonstrou doença na alça aferente do reservatório (Figura 2). Os demais tiveram suas ileostomias fechadas.

\begin{tabular}{|lll|}
\hline Doente & Características da DC & Tratamento realizado \\
\hline 1 & Fístulas perineais/perianais & Ileostomia \\
& + fístula reservatório-vaginal & \\
3 & Fístula reservatório-vaginal & Avanço mucoso \\
4 & Recidiva em íleo & Enterectomia \\
& Fístulas perineais/perianais & Ileostomia + \\
5 & + recidiva em íleo & enterectomia \\
& Fístulas perineais/perianais & Ileostomia + \\
6 & & ressecção do reservatório \\
7 & Recidiva em reservatório & Clínico \\
8 & Recidiva em reservatório & Clínico \\
9 & Recidiva em reservatório & Clínico \\
10 & Abscesso + recidiva em íleo & Não fechou ileostomia \\
11 & Recidiva em íleo & Clínico \\
\hline
\end{tabular}

FIGURA 2. Características da doença de Crohn e tratamento realizado
No seguimento tardio, três deles apresentaram fístulas perianais e perineais complexas, após 6, 18 e 80 meses da cirurgia de reservatório ileal, sendo que uma delas também evoluiu com fístula reservatório-vaginal. Todos necessitaram de nova derivação, com cicatrização das fístulas em dois deles. Um manteve supuração perianal apesar da ileostomia e foi necessária a excisão do reservatório. A doente com diagnóstico de DC no espécime da retocolectomia, apresentou fistula do reservatório para o intróito vaginal após 1 ano e 2 meses do fechamento da ileostomia. O tratamento proposto foi cirurgia de avanço mucoso, com resolução da fístula (Figura 2).

Houve recidiva da doença no íleo proximal em outros três doentes, sendo um deles o que apresentou múltiplas fístulas perineais e perianais. Este doente apresentou prolapso da ileostomia, com mucosa irregular e com erosões, sendo realizada enterectomia deste segmento 37 meses após a nova derivação intestinal e o estudo anatomopatológico confirmou DC. O segundo foi em um doente cujo diagnóstico da DC foi na proctectomia e a recidiva foi verificada na radiografia contrastada do intestino delgado, realizada 17 meses após fechamento da ileostomia. O terceiro evoluiu com suboclusão intestinal, sendo submetido a laparotomia 12 meses após fechamento do estoma. Durante a cirurgia foi observada estenose no íleo distal e outra área de calibre reduzido próximo ao reservatório ileal, sendo realizada ressecção do primeiro segmento, cujo estudo anatomopatológico foi compatível com DC, e dilatação do segundo, com ileostomia de proteção, fechada após 14 meses. Em outros três doentes, o exame endoscópico realizado devido à piora da função, após 16, 42 e 72 meses da confecção do reservatório ileal, demonstrou presença de inflamação no reservatório e as biopsias revelaram $\mathrm{DC}$ em dois deles e no outro, processo inflamatório inespecífico, havendo melhora clínica com tratamento medicamentoso (Figura 2).

O único doente que ainda não apresentou manifestação clínica da DC foi um dos dois que tiveram diagnóstico no espécime da proctectomia, com seguimento de 57 meses da cirurgia de reservatório ileal.

Atualmente, com acompanhamento médio de 76,5 meses, variando de 22 a 155 do diagnóstico da DC, todos estão com a doença controlada. Cinco doentes não fazem uso de medicação, três estão utilizando mesalazina, sendo que dois destes recebem também aplicação de infliximabe e, três outros, utilizam azatioprina, um deles em associação com infliximabe. Quatro doentes estão com derivação, sendo que o reservatório foi retirado em um deles e sete estão com os reservatórios ileais funcionantes, estes apresentando 6-10 evacuações ao dia e com continência praticamente normal.

\section{DISCUSSÃO}

Retocolectomia é largamente aceita como procedimento cirúrgico para RCUI e proporciona qualidade de vida satisfatória para a maioria dos doentes operados ${ }^{(23)}$. Entretanto, sua indicação na DC é questionável, havendo mais autores que a contra-indicam do que a defendem. Em várias séries de reservatórios ileais relatados na literatura, entre 2,5\%-13\% apresentam-se com 
diagnóstico posterior de $\mathrm{DC}^{(5,6,10,18,21)}$. Essa variação pode ser explicada pelos diferentes critérios diagnósticos da DC, ou seja, histopatológico, endoscópico ou apresentação clínica, além do tempo de seguimento desses doentes.

No entanto, o diagnóstico diferencial entre RCUI e DC nem sempre é fácil. Em 10\%-20\% das doenças inflamatórias intestinais acometendo o cólon e reto, não é possível fazer essa diferenciação clínica, endoscópica ou histológica, sendo esses casos denominados como colite indeterminada (CI). Vários trabalhos têm documentado eventual diagnóstico de DC em doentes com $\mathrm{CI}^{(1,11,13)}$. KOLTUN et al. ${ }^{(12)}$ verificaram que o diagnóstico de CI predispõe a complicações perineais e falência do reservatório ileal. MARCELO et al. ${ }^{(13)} \mathrm{e} \mathrm{YU} \mathrm{et} \mathrm{al.}{ }^{(25)}$ demonstraram que doentes com CI têm, significantemente, maior chance de desenvolver DC do que aqueles com RCUI, respectivamente, de $13 \%$ e $3 \%$, e $15 \%$ e $2 \%$.

O diagnóstico diferencial entre RCUI e DC é particularmente difícil em doentes com intensa inflamação aguda. Sete dos 112 pacientes de OCA et al. ${ }^{(4)}$ apresentavam diagnóstico de RCUI grave na colectomia, mas a subseqüente análise da peça da proctectomia revelou DC com achado de agregados linfóides, granulomas e envolvimento transmural do reto, além de áreas normais neste segmento.

Na presente casuística, 5 dos 11 doentes tinham sido operados por megacólon tóxico, o que poderia explicar a taxa de DC de $14,5 \%$, pouco maior que a referida na literatura. Dois dos diagnósticos de DC também foram obtidos a partir do espécime da proctectomia. Portanto, seria prudente, em doentes operados por megacólon tóxico, a biopsia do reto antes de se proceder à confecção do reservatório ileal.

A percentagem de diagnóstico tardio de DC de PEYREGNE et al. ${ }^{(18)}$ foi de $13 \%$, semelhante a desta série, sendo que dos sete doentes, três também foram operados por megacólon tóxico. $\mathrm{Na}$ evolução, três necessitaram de nova ileostomia e excisão do reservatório foi realizada em um deles.

Reservatórios ileais com DC podem apresentar complicações como fístulas, sepse pélvica, pouchitis secundária à DC e estenoses, mas o resultado desses doentes não é necessariamente ruim e muitos podem ter função satisfatória do reservatório. Morbidade e resultados à longo prazo são controversos. Alguns autores têm mostrado significante morbidade e falência do reservatório ${ }^{(2,3,5,7,8,10)}$. Entretanto, outros têm demonstrado que resultados funcionais a longo prazo nos doentes em que o reservatório é mantido depois do diagnóstico de DC, são similares aos da RCUI ${ }^{(4,6,15,21)}$. Também REGIMBEAU et al. ${ }^{(20)}$ e HARTLEY et al. ${ }^{(9)}$ verificaram bons resultados a longo prazo nos doentes com diagnóstico tardio de $\mathrm{DC}$ em reservatórios ileais.

TEKKIS et al. ${ }^{(22)}$ compararam um grupo de doentes com CI ou CI favorecendo o diagnóstico de RCUI e outro grupo com DC ou CI favorecendo diagnóstico de DC e verificaram falência do reservatório em $11,5 \%$ e $57,5 \%$, respectivamente. Entretanto, não observaram diferença em relação à ocorrência de sepse pélvica, estenose da anastomose, obstrução intestinal ou pouchitis.
REESE et al. ${ }^{(19)}$, analisando 10 estudos envolvendo um total de 3.103 doentes, verificaram que aqueles com reservatório ileal e DC, apresentaram significantemente mais estenoses da anastomose e mais falência do reservatório do que aqueles com RCUI ou CI. O mesmo foi verificado por BROWN et al. ${ }^{(3)}$, com taxas de complicações de $64 \%$ para DC, $43 \%$ para CI e $22 \%$ para RCUI. Além disso, $56 \%$ dos doentes com DC tiveram reservatórios disfuncionalizados ou excisados, comparados com $10 \%$ da CI e $6 \%$ da RCUI.

MYLONAKIS et al. ${ }^{(14)}$ compararam as complicações de doentes com DC sem envolvimento do intestino delgado ou região perianal submetidos a cirurgia de reservatório ileal e ileorreto anastomose. Num seguimento de mais de 10 anos, verificaram piores resultados no grupo dos reservatórios ileais, especialmente quando a doença foi diagnosticada em conseqüência das complicações. Nesse grupo, a taxa de excisão do reservatório foi de $47,8 \%$, realização de estoma proximal de $4,3 \%$ e média de ressecção intestinal de $65 \mathrm{~cm}$, comparada com $8 \%, 3 \%$ e $15 \mathrm{~cm}$ da anastomose ileorretal.

A taxa de falência de reservatório ileal com DC é referida entre $10 \%-57 \% \%^{(7,10,15,21,22)}$ e de excisão de $10 \%-53 \%^{(5,8,10,15,17,21,22)}$. Nesta série foi, respectivamente, de $36,4 \%$ e $9,1 \%$. As principais complicações observadas foram as fístulas relacionadas ao reservatório ileal, que foram verificadas em 36,4\% dos doentes, semelhantes ao relatado na literatura. Na maioria das vezes, as fístulas cicatrizaram com nova derivação intestinal e a excisão do reservatório foi necessária apenas em um caso. Com o surgimento de novas drogas no tratamento da DC, como os medicamentos biológicos, novas perspectivas surgem e a retirada definitiva do reservatório só estaria indicada na persistência da sepse pélvica.

DC envolvendo reservatório ileal, anastomose ileoanal ou íleo proximal tende a ser de difícil controle com alta morbidade relacionada a complicações como abscessos, fístulas e estenoses. Estes doentes geralmente requerem tratamento medicamentoso agressivo com combinação de salicilatos, esteróides, agentes imunossupressores e medicamentos biológicos, como o infliximabe.

\section{CONCLUSÃO}

Embora não haja consenso na literatura, não é rotina do Serviço de Proctologia em que este trabalho se desenvolveu, a realização da cirurgia de reservatório ileal em doentes com diagnóstico ou suspeita de DC, mesmo na ausência de envolvimento do intestino delgado ou acometimento perianal. $\mathrm{Na}$ presença de características da DC, uma colectomia preliminar poderia ser realizada para estudo de todo o espécime cirúrgico, além de biopsias do reto remanescente naqueles operados por megacólon tóxico. Doentes operados de reservatórios ileais como RCUI e com diagnóstico posterior de DC estão sujeitos a complicações como abscessos, fístulas, sepse pélvica, pouchitis e estenoses, porém, reservatórios ileais com DC podem ter função satisfatória e devem ser mantidos. 
Ayrizono MLS, Meirelles LR, Leal RF, Coy CSR, Fagundes JJ, Góes JRN. Long-term outcomes of ileal pouch after secondary diagnosis of Crohn's disease. Arq Gastroenterol. 2008;45(3):204-7.

ABSTRACT - Background - Total rectocolectomy and ileal pouch-anal anastomosis is the choice surgical procedure for patients with ulcerative colitis. In cases of Crohn's disease post-operative diagnosis, it can be followed by pouch failure. Aim - To evaluate ileal pouch-anal anastomosis long-term outcome in patients with Crohn's disease. Methods - Between February 1983 and March 2007, 151 patients were submitted to ileal pouch-anal anastomosis by Campinas State University Colorectal Unit, Campinas, SP, Brazil, 76 had pre-operative ulcerative colitis diagnosis and 11 had post-operative Crohn's disease diagnosis. Crohn's disease diagnosis was made by histopathological biopsies in nine cases, being one in surgical specimen, two cases in rectal stump, small bowel in two cases, ileal pouch in three and in perianal abscess in one of them. The median age was 30.6 years and eight (72.7\%) were female. Results - All patients had previous ulcerative colitis diagnosis and in five cases emergency colectomy was done by toxic megacolon. The mean time until of Crohn's disease diagnosis was 30.6 (6-80) months after ileal pouch-anal anastomosis. Ileostomy closure was possible in 10 cases except in one that had ileal pouch fistula, perianal disease and small bowel involvement. In the long-term follow-up, three patients had perineal fistulas and one had also a pouch-vaginal fistula. All of them were submitted to a new ileostomy and one had the pouch excised. Another patient presented pouch-vaginal fistula which was successfully treated by mucosal flap. Three patients had small bowel involvement and three others, pouch involvement. All improved with medical treatment. Presently, the mean follow-up is 76.5 months and all patients are in clinical remission, and four have fecal diversion. The remaining patients have good functional results with 6-10 bowel movements/day. Conclusion - Crohn's disease diagnosis after ileal pouch-anal anastomosis for ulcerative colitis may be usual and later complications such fistulas and stenosis are common. However, when left in situ ileal pouch is associated with good function.

HEADINGS - Crohn disease. Proctocolitis. Colonic pouches. Ileal pouches.

\section{REFERÊNCIAS}

1. Bodzin JH, Klein SN, Priest SG. Ileoproctostomy is preferred over ileoanal pullthrough in patients with indeterminate colitis. Am Surg. 1995;61:590-3.

2. Braveman JM, Schoetz DJ, Marcello PW, Roberts PL, Coller JA, Murray JJ, Rusin LC. The fate of the ileal pouch in patients developing Crohn's disease. Dis Colon Rectum. 2004;47:1613-9.

3. Brown CJ, Maclean AR, Cohen Z, Macrae HM, O'Connor BI, McLeod RS. Crohn's disease and indeterminate colitis and the ileal pouch-anal anastomosis: outcomes and patterns of failure. Dis Colon Rectum. 2005;48:1542-9.

4. de Oca J, Sánchez-Santos R, Ragué JM, Biondo S, Pares D, Osorio A, del Rio C, Jaurrieta E. Long-term results of ileal pouch-anal anastomosis in Crohn's disease. Inflamm Bowel Dis. 2003;9:171-5.

5. Deutsch AA, McLeod RS, Cullen J, Cohen Z. Results of the pelvic-pouch procedure in patients with Crohn's disease. Dis Colon Rectum. 1991;34:475-7.

6. Fazio VW, Ziv Y, Church JM, Oakley JR, Lavery IC, Milson JW, Schroeder TK. Ileal pouch-anal anastomoses complications and function in 1005 patients. Ann Surg. 1995;222:120-7.

7. Foley EF, Schoetz DJ Jr, Roberts PL, Marcello PW, Murray JJ, Coller JA, Veidenheimer MC. Rediversion after ileal pouch-anal anastomosis. Causes of failure and predictors of subsequent pouch salvage. Dis Colon Rectum. 1995;38:793-8.

8. Grobler SP, Hosie KB, Affie E, Thompson H, Keighley MRB. Outcome of restorative proctocolectomy when the diagnosis is suggestive of Crohn's disease. Gut. 1993;34:1384-8.

9. Hartley JE, Fazio VW, Remzi FH, Lavery IC, Church JM, Strong SA, Hull TL, Senagore AJ, Delaney CP. Analysis of the outcome of ileal pouch-anal anastomosis in patients with Crohn's disease. Dis Colon Rectum. 2004;47:1808-15.

10. Hyman NH, Fazio VW, Tuckson WB, Lavery IC. Consequences of ileal pouch-anal anastomosis for Crohn's colitis. Dis Colon Rectum. 1991;34:653-7.

11. Kangas E, Matikainen M, Mattila J. Is "indeterminate colitis" Crohn's disease in the long-term follow-up? Int Surg. 1994;79:120-3.

12. Koltun WA, Schoetz DJ Jr, Roberts PL, Murray JJ, Coller JA, Veidenheimer MC Indeterminate colitis predisposes to perineal complications after ileal pouch-anal anastomosis. Dis Colon Rectum. 1991;34:857-60.
13. Marcello PW, Schoetz DJ Jr, Roberts PL, Murray JJ, Coller JA, Rusin LC, Veidenheimer MC. Evolutionary changes in the pathologic diagnosis after the ileoanal pouch procedure. Dis Colon Rectum. 1997;40:263-9.

14. Mylonakis E, Allan RN, Keighley MR. How does pouch construction for a final diagnosis of Crohn's disease compare with ileoproctostomy for established Crohn's proctocolitis? Dis Colon Rectum. 2001;44:1137-43.

15. Panis Y, Poupard B, Nemeth J, Lavergne A, Hautefeuille P, Valleur P. Ileal pouch/anal anastomosis for Crohn's disease. Lancet. 1996;347:854-7.

16. Parks AG, Nicholls RJ. Proctocolectomy without ileostomy for ulcerative colitis. $\mathrm{Br}$ Med J. 1978;2:85-8

17. Paye F, Penna C, Chiche L, Tiret E, Frileux P, Parc R. Pouch-related fistula following restorative proctocolectomy. Br J Surg. 1996;83:1574-7.

18. Peyregne V, Francois Y, Gilly FN, Descos JL, Flourie B, Vignal J. Outcome of ileal pouch after secondary diagnosis of Crohn's disease. Int J Colorectal Dis. 2000;15:49-53.

19. Reese GE, Lovegrove RE, Tilney HS, Yamamoto T, Heriot AG, Fazio VW, Tekkis PP. The effect of Crohn's disease on outcomes after restorative proctocolectomy. Dis Colon Rectum. 2007;50:239-50.

20. Regimbeau JM, Panis Y, Pocard M, Bouhnik Y, Lavergne-Slove A, Rufat P, Matuchansky C, Valleur P. Long-term results of ileal pouch-anal anastomosis for colorectal Crohn's disease. Dis Colon Rectum. 2001;44:769-78.

21. Sagar PM, Dozois RR, Wolff BG. Long-term results of ileal pouch-anal anastomosis in patients with Crohn's disease. Dis Colon Rectum. 1996;39:893-8.

22. Tekkis PP, Heriot AG, Smith O, Smith JJ, Windsor AC, Nicholls RJ. Long-term outcomes of restorative proctocolectomy for Crohn's disease and indeterminate colitis. Colorectal Dis. 2005; 7:218-23

23. Tiainen J, Matikainen M. Health-related quality of life after ileal J pouch-anal anastomosis for ulcerative colitis: long-term results. Scand J Gastroenterol. 1999;34:601-5.

24. Warren BF, Shepherd NA. The role of pathology in pelvic ileal reservoir surgery. Int J Colorectal Dis. 1992; 7:68-75.

25. Yu CS, Pemberton JH, Larson D. Ileal pouch-anal anastomosis in patients with indeterminate colitis. Long-term results. Dis Colon Rectum. 2000;43:1487-96.

Recebido em 23/7/2007. Aprovado em 18/1/2008 